\title{
ECMO Challenges and Its Future: Indian Scenario
}

\author{
Suneel Pooboni ${ }^{1}$ Venkat Goyal ${ }^{2}$ Pranay Oza ${ }^{3}$ Poonam Malhotra Kapoor ${ }^{4}$ \\ ${ }^{1}$ Pediatric Critical Care and ECMO Unit, University Hospitals of \\ Leicester, Leicester, United Kingdom \\ 2Department of Cardiology, RVCC, Mumbai, Maharashtra, India \\ 3 Intensivist and ECMO Specialist, RVCC, Mumbai, Maharashtra, India \\ ${ }^{4}$ Department of Cardiac Anaesthesia, CTC, AlIMS, New Delhi, India \\ Address for correspondence Pranay Oza, BHMS, Intensivist and \\ ECMO Specialist, RVCC, Mumbai, Maharashtra, India \\ (e-mail: drpranay.oza@gmail.com). \\ J Card Crit Care TSS 2017;1:89-94
}

\begin{abstract}
History of extracorporeal membrane oxygenation (ECMO) in India dates back to 2001. Initially, ECMO was started for postoperative cardiac cases, but from 2008, it was introduced to intensive critical care unit as a regular practice for refractory cardiogenic shock and acute respiratory distress syndrome. Gradually, the utility of ECMO keeps on increasing such as in poisoning, transplant unit, and even in tracheobronchial surgery. Some centers even started using ECMO for extracorporeal cardiopulmonary resuscitation, even though the data is insufficient. In academic field also, ECMO has had great progress in India with three books and numerous paper presentations by Indian authors, regular conferences, and continued medical education. There were lot of challenges in establishing ECMO in Indian scenario which were addressed successfully; a few challenges are still there which need to be addressed pre-emptively. The most common challenges of ECMO were awareness, cost, initiation at right time, outcome, nosocomial infections, and transport. Transport of critically ill patient, especially on

Keywords

- ECMO future

- ECMO India

- ECMO challenges

- ECMO journey ECMO, is risky and also involves lots of cost. To overcome this problem, flying squad was established in 2010 where the team goes to the hospital where the patient is and initiates and manages ECMO there itself. The future of ECMO in India is quite promising, not only with increasing number of cases and centers, but also in terms of costeffectiveness and technology.
\end{abstract}

\section{Introduction}

Extracorporeal membrane oxygenation (ECMO) is a technique developed to ensure a prolonged but temporary support of heart or lung in patients suffering from reversible cardiac and/or respiratory failure using mechanical assist device. ${ }^{1}$ ECMO helps in stabilizing the patient and sustains life till the time the heart and lung recover. In addition, it also gives rest to the organ which ultimately helps in faster recovery. In irreversible diseases, it can act as a bridge for device therapy or transplant. ${ }^{2}$

It can be utilized for wider range of indications for refractory heart or lung failure or cardiopulmonary failure, when maximal conventional management is failed. In addition to regular use for acute respiratory or cardiac failure, it can also be used for perioperative stabilization and in transplant intensive care unit (ICU). Being a reasonably expensive procedure with significant complications with inadvertent use, it is under-utilized in Indian scenario.

\section{Journey of ECMO in India}

History of ECMO in India dates back to 2001. Initially, ECMO was started for postoperative cardiac cases, especially in All India Institute of Medical Sciences (AIIMS), Delhi, and later in Narayana Hrudayalaya, Bangalore, in 2004. For the first few years, only anecdotal cases have been reported for using ECMO for reasons other than cardiac surgical cases. ECMO was introduced to intensive critical care unit as a regular practice for refractory cardiogenic shock and acute respiratory distress syndrome (ARDS) from 2008. Riddhivinayak Critical Care and Cardiac Center (RVCC), Mumbai, remains the pioneer in bringing this power of science with its complete utility in the field of critical care for both adult and pediatric.
DoI https://doi.org/ 10.1055/s-0038-1626672 ISSN 2457-0206.
Copyright @ 2017 Official Publication of The Simulation Society (TSS), accredited by International Society of Cardiovascular Ultrasound (ISCU)
License terms

() (1) $\ominus \circledast$ 
In 2010, the flying ECMO squad was established, comprising a team of four people including staff nurse, perfusionist, and ECMO physician. The team used to fly to different cities of India for initiating ECMO and managing patients on ECMO in respective centers. The team stays there till the time the patient is decannulated. This model was initiated to create awareness about ECMO, train the local teams, and give the benefit of ECMO to the entire country. The flying ECMO squad from RVCC has performed ECMO in around 20 major cities and in 58 tertiary care hospitals till today.

In later part of 2011, ECMO was started in transplant ICU for pre-, during, and post-lung transplant patients. In 2012, the first long-distance transport on ECMO $(\sim 250 \mathrm{~km})$ by road was performed. In the later part of 2013, the practical application of ECMO was extended for treating selective toxidromes. Dr. Vivek Gupta and team from Hero DMC Heart Institute, Ludhiana, started using ECMO regularly for aluminum phosphide (celphos) poisoning cases. They have worked on more than 50 patients and demonstrated survival rate of around $60 \%$ to $70 \%$.

Apart from the clinical applications, the teaching and training aspects of ECMO have seen a new era of development since 2010. ECMO Society of India was established in November, 2010, with an intention of developing and sharing this novel technology in India and get all those who are doing ECMO under one roof. Very soon, neighboring countries joined the training program to reap the benefits.

First national conference on ECMO was held on 27 and 28 November, 2010 by ECMO Society of India. Since then, it is conducted in the month of November every year. It has been customary to host ECMO training courses of 5 days in summer, in the month of June at RVCC in Mumbai and in winter as per the training demands.

South and West Asia chapter of extracorporeal life support organization (ELSO) was established in the year 2013, with the main intention being to support and improve understanding and practice of extracorporeal life support systems in the member countries.

\section{ECMO the Current Trend}

We progressed from 2001 till date and especially in the past 5 years. The numbers of ECMO centers are increasing in India. As per the anonymous data obtained from two companies dealing with the sales of ECMO machines, around 160 ECMO machines were sold in the past two years in India and approximately 700 circuits in the last year. There are three more companies which deal with ECMO equipment but are not very active; hence, their data are still missing. As per the anonymous data obtained from the dealers of ECMO equipment and personal communications, around 54 centers are actively doing ECMO in India.

Currently, the common indication for ECMO in Indian scenario is ARDS (especially H1N1 and mainly in adults), poisoning (especially aluminum phosphide poisoning), cardiac ECMO (postcardiac surgery especially in pediatric and ischemic cardiogenic shock), and for pre-and post-transplant management. The other indications where ECMO has also been used in India are extracorporeal cardiopulmonary resuscitation (ECPR) and elective ECMO for complex tracheal and airways procedure. Neonatal ECMO is sparingly used for indications such as meconium aspiration syndrome and congenital diaphragmatic hernia. Cost factors could be a reason for this. ECMO is under-utilized for other indications such as severe septic shock, donor preservation, and organ harvesting (extracorporeal interval support for organ retrieval).

In the academic field, there is satisfactory progress. Three books were published in the country along with numerous papers on ECMO. The first book on ECMO was published during the first annual conference of ECMO Society of India. The book was named ECMO Practical Manual Volume I and was written by Dr. Venkat Goyal and Dr. Pranay Oza, which covered the practical aspects of ECMO. More and more people from relevant disciplines are showing their interest and involvement in learning ECMO and making it their carrier choice. Annual conference and annual training course in the month of November and June respectively are being organized by ECMO Society of India. There are many regional conferences, training programs, and continued medical education activities being organized at local levels. In addition, to share their experiences and for help in difficult case scenarios, society has its Facebook account, Google group, and WhatsApp group.

\section{ECMO Challenges}

The challenges in the practice of ECMO in India are numerous. Awareness of the efficacy and right indicators for ECMO, choosing the right equipment, travel delays during transport, cost factors, and availability of financial resources are a few of them. We could find solutions to some of these problems, while the others need to be addressed proactively.

Awareness among the medical fraternity and public education is essential to initiate ECMO at the right time. Regular training programs are being conducted at regional levels. Awareness among physicians, intensivists, and pediatricians is improving, but public education is still an issue. Now more physicians are thinking about ECMO much earlier at least for indications such as acute respiratory failure, especially in H1N1 infections.

Currently, three major distributors are actively marketing ECMO equipment and trying to address the issues related to equipment. The availability of Avalon double lumen cannula (MAQUET Holding B.V. \& Co. KG.) for respiratory ECMO is still an issue. But nowadays, Origen double lumen cannula (OriGen Biomedical) availability is improving. Cost factors and importing licenses are some of the compounding problems. Ideal solution for this issue would be establishing local manufacturing of suitable ECMO equipment and cannulae within the country. This initiative should be helpful in lowering the costs.

Transporting a patient while on extracorporeal life support is a complex challenging endeavor. ${ }^{3}$ Critical care transport is getting established by road for limited distance and there 
are a few cases of ECMO road transports that have been reported. But the ECMO air transport from one state to other states of India will involve considerable costs and only anecdotal cases have been reported. Transport of critically ill patients puts them at increased risk of significant morbidity and mortality. ${ }^{4}$ As a result, the transport of ECMO (mobile ECMO) patients in India remains a real challenge as there are a very few ECMO centers, the huge area and large population of the country, and the diversities of available resources between urban and rural areas. Road transportation is the most common mode of our ECMO transfers. It is cheaper but its limitations are traffic congestion, extreme road conditions, and travel times. The maximum distance covered is up to 250 miles, ${ }^{5}$ corresponding to almost 5 to 6 hours of travel time. Air transportation is a good option for long distances but is costly. ${ }^{6}$

To overcome these difficulties, flying ECMO squad was established and now there are two centers in India (namely, RVCC, Mumbai, and Unique Hospital, Surat) which have around six to seven teams. Each flying squad, consisting of three to four team members, can go to hospitals where the patient needs ECMO. They initiate ECMO and stay there till the time patient is decannulated.

Nosocomial drug resistant infection is one of the major problems contributing to reduction in the survival rate following ECMO therapy in our country. ${ }^{7}$ Onset of nosocomial infections has been responsible for $20 \%$ reduction in survival rate following ECMO. Not only does it increase mortality, but the morbidity will also be extended by at least 1 additional week of ECMO run, ICU stay, and the comorbid complications secondary to drug-resistant infections. ${ }^{7}$ Universal aseptic precautions, staff education, minimizing usage of broad spectrum antibiotics, and de-escalating the antibiotics when not needed are extremely important steps in curtailing the emergence of drug resistance.

Monitoring of anticoagulation is usually done by measuring activated clotting time and activated partial thromboplastin time. Monitoring by antifactor Xa is not a routine practice. In a long-term ECMO, major or minor bleeding remains a major issue. Mortality related to bleeding is around $8 \%$ to $10 \%$.

The major hindrance in the development of ECMO program in India is the cost factor. ECMO treatment becomes costlier because of the high cost of disposables, higher recurring or maintenance charges per day, increased length of ICU stay, need for higher antibiotics and blood products (if required), and involvement of multiple professionals. In the current scenario, ECMO services are being offered at a few private sector multispecialty hospitals, where most of the cost is borne by the family of the patient. Patients' families might get some help from corporate health insurance policies or company insurance schemes. In current times, a few of the corporates and mediclaim policies have started partially or fully sponsoring ECMO expenses, but there is still a long way to go.

We wish for more and more government-funded/ autonomous institutions to adopt this technology in a cost-effective way. With the help of bulk purchasing, the cost of disposables can be brought down. The additional advantages at government-funded tertiary care center are that manpower, maintenance cost, and blood products are not going to be an additional cost. So, it can easily be made cost-effective in government sector but one requires a dedicated person to do it.

To know the prognosis, evaluating variables such as the best time for initiation and formulate guidelines, we need data of the cases performed. In the current scenario, we are getting all required data and guidelines from ELSO. We suspect that there might be a lot of regional variations in terms of indications, available technology, and economic status. ECMO Society of India and Indian Network of Specialist Pediatric Intensivists for Research Education and Data (INSPIRED) group of intensive care subchapter of Indian Academy of Pediatrics are encouraging voluntary reporting of the data. At present, we have center-specific data (which is confidential), data from INSPIRED group (only pediatric ECMO data), and data collected by ECMO Society of India from a few voluntary centers. Reporting of ECMO data in India is grossly inadequate.

INSPIRED group has collected data from nine different centers who were performing pediatric ECMO for the period of January 2011 to January 2017. Total of 86 cases of ECMO were reported, out of which 52 children underwent respiratory ECMO with $40 \%$ survival and 34 children underwent cardiac ECMO with $73.5 \%$ survival.

ECMO Society of India has collected data from volunteer centers and also taken some data published in INSPIRED group. This is the data of around 18 centers from January 2010 to January 2017, actively doing ECMO in India and includes both pediatric and adult data. As per this data, cardiac ECMO cases are 295 with survival of $57.62 \%$, respiratory ECMO cases are 307 with survival of $41.37 \%$, and ECPR cases are 18 with survival of $22.22 \%$. So, the total 620 cases of ECMO reported with survival of $48.55 \%$.

This data obtained is much less when compared with the data obtained from two companies of installation of $160 \mathrm{ma}-$ chines and selling of 700 circuits in last year.

\section{Integrated ECMO: AlIMS Experience}

ECMO equipment may be modified as per the specific needs of the patient and the operating personnel. The integrated ECMO-CPB circuit devised at the author's institute has been instrumental in the development and progress of the institute's ECMO program and has helped in improving the survival of patients. The integrated ECMO-CPB circuit involves a slight modification of the cardiopulmonary bypass (CPB) circuit by utilizing the ECMO oxygenator during the $\mathrm{CPB}$. The oxygenator that is routinely a part of CPB circuit is not used because of its short life.

Advantages of the redesigned venoarterial integrated ECMO are so as to:

1. Incorporate $\mathrm{CPB}$ circuit

2. Increase preparedness/availability

3. Cut-down assembly time

4. Reduce possibility of cardiopulmonary resuscitation

5. Cost-effectiveness 
In this article, we analyze the survival after ECMO support in children undergoing repair of congenital heart disease (CHD) over a 15-year period, with reference to the indication for ECMO support, time of initiation of ECMO support, underlying CHD, and the effect of use of integrated ECMOCPB circuit. Survival was considered if the child survived till discharge from the ICU ( - Table $\mathbf{1}$ ).

Another big challenge is the ethical dilemma among the critical care specialist and ECMO specialist. In present scenario, it is a complex picture. At one end you have better survival chances with ECMO, but at the same time the outcome is not guaranteed, health care is self-sponsored, and is an expensive therapy. With all this background, it becomes very difficult to decide whether to offer this modality to a middle-class family or not. Second dilemma is consideration of stopping life support (ECMO) in case of lack of response to prolonged duration on ECMO, especially when there are financial issues. There is also a limitation of ECMO machines and trained manpower running ECMO centers. With this limitation, especially during epidemics (e.g., H1N1 epidemic), it becomes difficult to prioritize the patient for ECMO. Solution to these dilemmas are well-set guidelines and protocols, a team decision (rather than individual), and good family counseling.

\section{ECMO Future: Indian Scenario}

The future of ECMO in general and especially in India looks quite promising. The brighter picture is not only in the increased number of ECMO cases and increased number of ECMO centers, but also with the better and cost-effective ECMO technology. In one of the market surveyed poised, the ECMO machine market will reach USD 305.3 million by 2021 from USD 247.2 million in $2016 .^{8}$

The mean market size for ECMO in India would be 70.55 million in 1 year. The reason for this jump is multifactorial. With the existing awareness, better outcome, and huge number of patients requiring ECMO therapy, eventually all tertiary care hospitals will have one or more ECMO units. There will be increased indications of ECMO. ECMO will be utilized for perioperative and periprocedural stability. There will be more numbers of cardiac ECMO for cardiogenic shock secondary to acute myocardial infarction. In severe ARDS, people will prefer to use ECMO and noninvasive ventilation rather than using invasive conventional ventilation. ECMO transport will be routine practice and will be more located state-wise or limited to cities as most of the cities will have ECMO units.
ECMO outcome will also improve and even ECMO run will be shorter as ECMO initiation will be early.

Looking at the huge potential, more companies are expected to launch ECMO products, which will lead to competitive market, thereby leading to decrease in cost. There is a good likelihood of manufacturing the components of ECMO and ECMO cannulae in India.

With increasing utility, better outcome, and safer technology, ECMO will soon become an established armamentarium in ICU settings. This will lead to the favorable reimbursement scenario. ECMO facility will also be available in tertiary government institute and in medical colleges.

ECMO Society of India has already started collecting Indian data (see - Table 2). Although it is still at a very primitive stage, in near future it will get much organized. So, we will have Indian ECMO data and also Indian guidelines from ECMO Society of India. The society is in the process of standardization, regulation, and monitoring the training program and delivery of ECMO services. ECMO Society of India is starting with fellowship and certificate course for ECMO for both medical and paramedical personnel (perfusionist, staff nurse, respiratory therapist).

There will be more papers and publications from India on various aspects of ECMO. India will play a leading role in establishing certain guidelines like guidelines for ECMO in poisoning and guidelines for ECMO in acute decompensated heart failure due to huge volume.

\section{Summary}

The scenario is rapidly changing in India, from experimental science (2007-2008), to modality that can be used as last resource (2010), to modality to be used when ventilation requirement is increasing (2012), and to the current scenario of using ECMO before ventilator (especially in swine flu).

Cost remains a major issue as in Indian scenario health is self-sponsored, but it should not be the limiting factor. Cost can be further decreased by using simpler machines, by assembling your own circuit, and by reducing the number of unnecessary investigations. More awareness, timely intervention, and more number of cases will get the cost down. What it needs is passion rather than anything else-a passion for saving a life when death is almost certain and a passion for constant striving for excellence. What it needs is change of vision. Rather than asking "Can India afford ECMO?," we should say "Make ECMO affordable for India."

Table 1 Outcome by time of initiation and use of integrated ECMO-CPB circuit

\begin{tabular}{|l|l|l|}
\hline Time of intervention & Survived $\boldsymbol{n}(\%)$ & Died $\boldsymbol{n}(\%)$ \\
\hline Integrated ECMO-CPB circuit & $144(76)$ & $45(24)$ \\
\hline Failure to wean from CPB & $30(45.45)$ & $36(54.55)$ \\
\hline Postoperative ECMO & $9(33)$ & $18(67)$ \\
\hline
\end{tabular}

Abbreviation: ECMO-CPB, extracorporeal membrane oxygenation-cardiopulmonary bypass. 


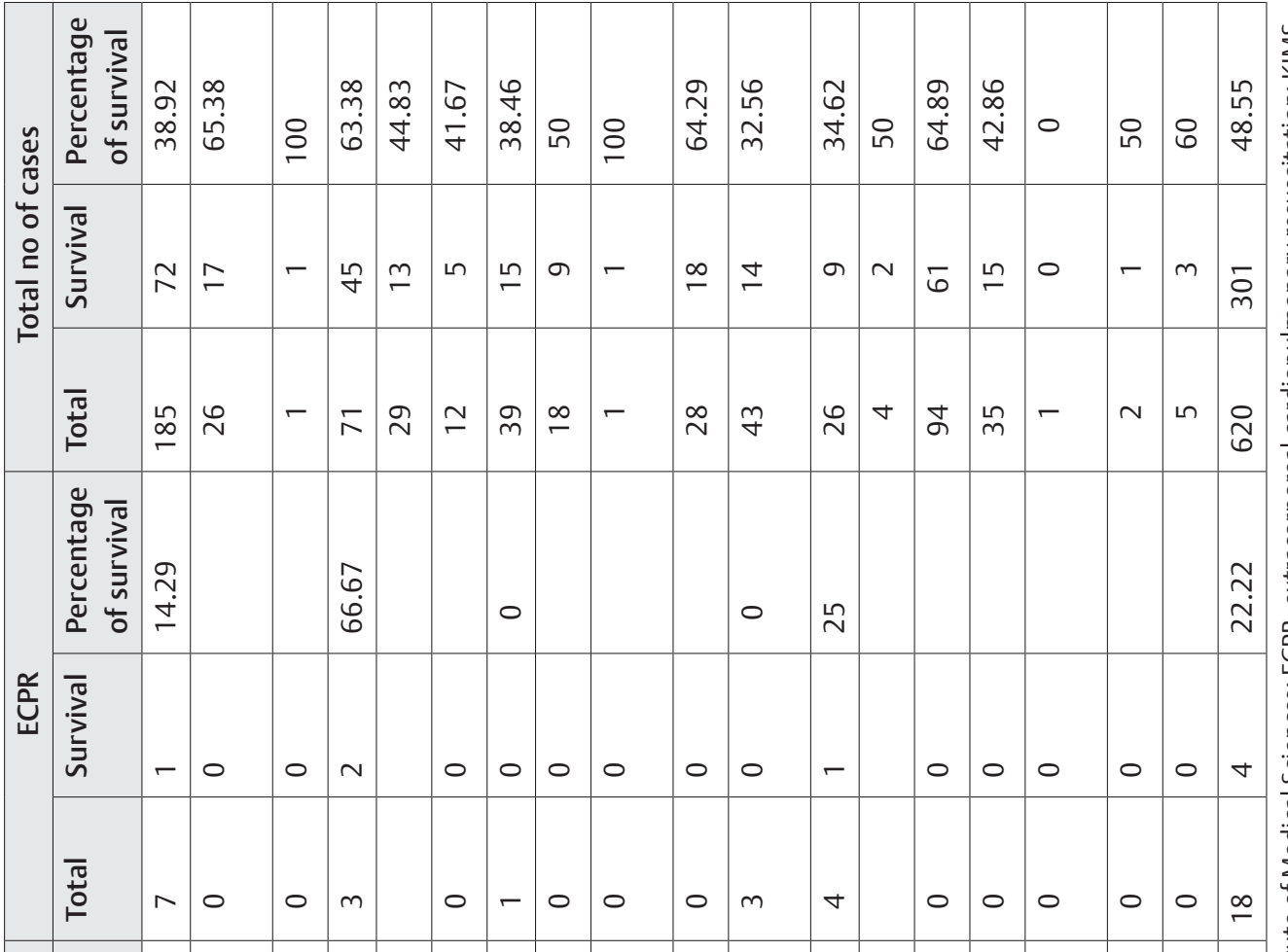

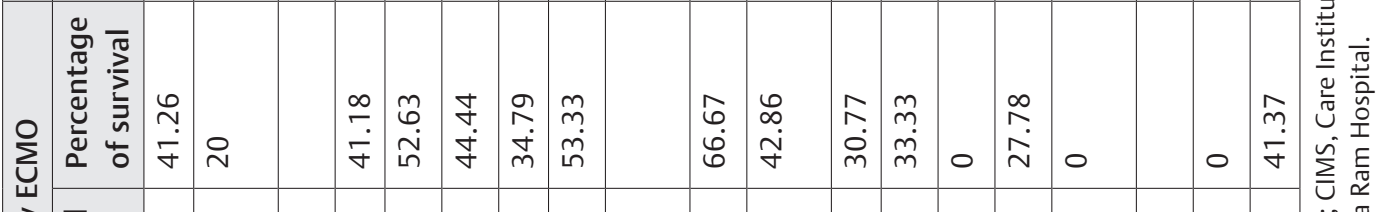

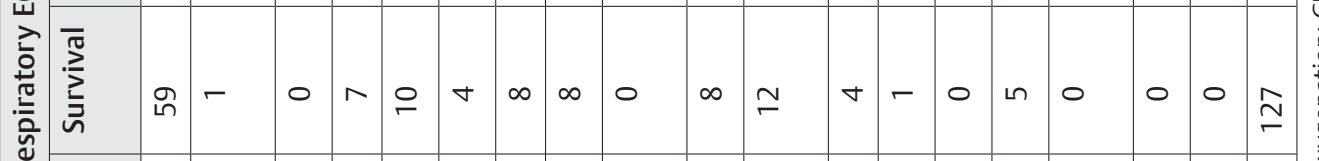

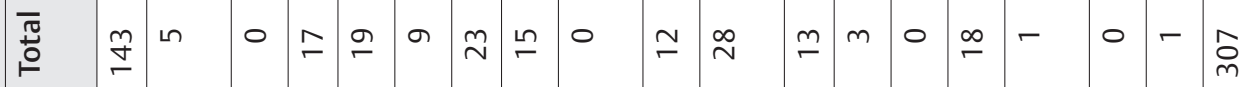

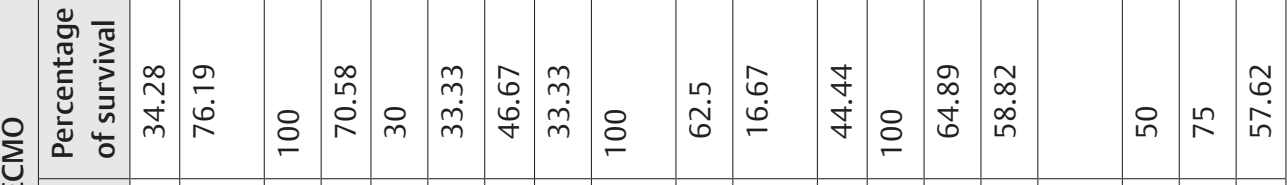

\begin{tabular}{|c|c|c|c|c|c|c|c|c|c|c|c|c|c|c|c|c|c|c|c|}
\hline 吝 & $\stackrel{\simeq}{ }$ & $\stackrel{6}{-2}$ & - & $\stackrel{m}{m}$ & $m$ & - & $r$ & - & - & $\stackrel{0}{\circ}$ & $\sim$ & $\nabla$ & - & $\overline{6}$ & $\stackrel{\circ}{\square}$ & 0 & - & $m$ & 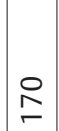 \\
\hline $\bar{\pi}$ & $\stackrel{\stackrel{n}{m}}{m}$ & $\bar{\sim}$ & - & in & $\circ$ & $m$ & $\stackrel{\llcorner}{\sim}$ & $m$ & - & $\stackrel{0}{\circ}$ & $\simeq$ & $\sigma$ & - & ন & $\approx$ & 0 & $\sim$ & $\nabla$ & $\stackrel{n}{2}$ \\
\hline
\end{tabular}

\begin{tabular}{|c|c|c|c|c|c|c|c|c|c|c|c|c|c|c|c|c|c|c|c|c|}
\hline & 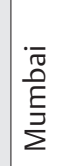 & 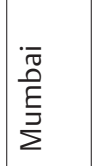 & 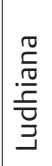 & 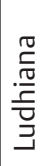 & 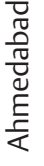 & 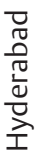 & $\begin{array}{l}\frac{\pi}{0} \\
\frac{\pi}{\pi} \\
\frac{\pi}{v} \\
\frac{\pi}{n}\end{array}$ & 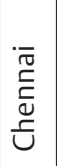 & 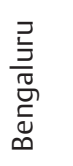 & 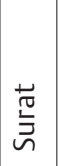 & $\begin{array}{l}\frac{\pi}{\tilde{0}} \\
\frac{\overrightarrow{0}}{\overline{0}} \\
\underline{\underline{0}}\end{array}$ & 容 & 穿 & 䆣 & $\stackrel{\overline{\bar{s}}}{\overline{\mathrm{g}}}$ & $\frac{\overline{\bar{E}}}{\overline{\mathrm{D}}}$ & $\frac{\pi}{\frac{\pi}{0}}$ & $\overline{\overline{\bar{s}}}$ & & 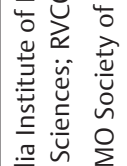 \\
\hline 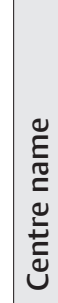 & $\underbrace{\breve{v}}_{\alpha}$ & 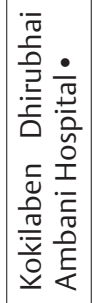 & $\dot{\tilde{n}}$ & $\sum_{0}$ & $\tilde{j}$ & 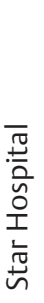 & $\sum_{\underline{\underline{y}}}^{n}$ & 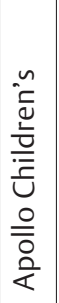 & 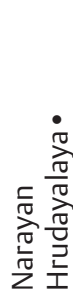 & 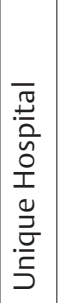 & 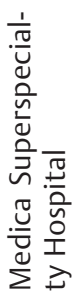 & $\cong$ & $\underset{\dot{\vec{y}}}{\dot{\dot{y}}}$ & $\varliminf_{\underline{\Sigma}}^{\underline{\Sigma}}$ & $\begin{array}{l}\frac{T}{\stackrel{v}{u}} \\
\text { n }\end{array}$ & 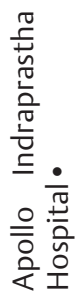 & 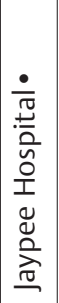 & 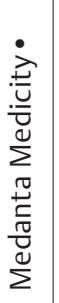 & $\begin{array}{l} \\
\frac{\pi}{0} \\
\stackrel{0}{\circ}\end{array}$ & 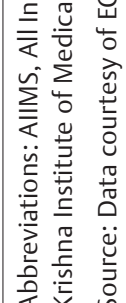 \\
\hline
\end{tabular}




\section{References}

1 Oza P, Goyal V. Basics of ECMO introduction. In: Oza P, Goyal $\mathrm{V}$, eds. ECMO - Theoretical Manual, 1st ed. ECMO Society of India; 2012;2:1-9

2 Malhotra P, Goyal V, Oza P. ECMO - a new therapeutic modality: where we are in India. In: Wander G, Pareek K, eds. Medicine Update 2016. Jaypee Brothers Medical Publishers, India; 2016;26:1634-1638

3 Oza P, Goyal V. Portable ECMO and transport. J Pediatr Crit Care 2017;4(2):82-92

4 Foley DS, Pranikoff T, Younger JG, et al. A review of 100 patients transported on extracorporeal life support. ASAIO J 2002;48(6):612-619
5 Dirnberger D, Fiser R, Harvey C, et al Guidelines for ECMO Transport. Available at https://www.elso.org/Portals/0/Files/ ELSO\%20GUIDELINES\%20FOR\%20ECMO\%20TRANSPORT_ May2015.pdf., Accessed January 16, 2018

6 Khurana H, Mehta Y, Dubey S. Air medical transportation in India: our experience. J Anaesthesiol Clin Pharmacol 2016;32(3):359-363

7 Pooboni S. ECMO: an Indian perspective, challenges and opportunities. J Pediatr Crit Care 2017;4:33-37

8 Patwardhan H. ECMO Machine Market By Application, Modality, and Region to 2022. www.wmcactionnews5.com [Online]. Accessed October 2, 2017 